Pacific Journal of Mathematics

ON REGULAR SUBDIRECT PRODUCTS OF SIMPLE ARTINIAN 


\title{
ON REGULAR SUBDIRECT PRODUCTS OF SIMPLE ARTINIAN RINGS
}

\author{
Chen-Lian Chuang and PJek-Hwee Lee
}

\begin{abstract}
We construct a counterexample to settle simultaneously the following questions all in the negative: (1) Is a regular subdirect product of simple artinian rings unit-regular? (2) If $R$ is a regular ring such that every nonzero ideal of $R$ contains a nonzero ideal of bounded index, is $R$ unit-regular? (3) Is a regular ring with a Hausdorff family of pseudo-rank functions unit-regular? (4) If $R$ is a regular ring which contains no infinite direct sum of nonzero pairwise isomorphic right ideals, is $R$ unit-regular? (5) Is a regular Schur ring unit-regular?
\end{abstract}

In [1] Goodearl proposed a list of open problems on regular rings. Some involve potential sufficient conditions for a regular ring to be unit-regular. The primary aim of this paper is to construct a counterexample for the questions 6, 7, 8, 9 (second part) and 11 in Goodearl's book.

Among others the sixth question asks: Is a regular subdirect product of simple artinian rings always unit-regular? In [4] Tyukavkin has shown that any regular algebra over an uncountable field, which is a subdirect product of countably many simple artinian rings, is unitregular. Recently, Goodearl and Menal [2] have generalized this result by showing that any regular algebra over an uncountable field, which has no uncountable direct sums of nonzero right or left ideals, must be unit-regular; in particular, any regular algebra over an uncountable field, which has a rank function, is unit-regular. In this paper we shall construct an example of a regular ring which is a subdirect product of countably many simple artinian rings but is not unit-regular.

Let $F$ be a countable field, $F[t]$ the ring of polynomials over $F$ in an indeterminate $t$, and $F(t)$ the quotient field of $F[t]$. Define an exponential valuation $\partial$ on $F(t)$ by $\partial r(t)=+\infty$ if $r(t)=0$ and $\partial r(t)=n$ if $r(t)=t^{n} f(t) / g(t)$ where $n$ is an integer and $f(t), g(t) \in$ $F[t]$ with $t \nmid f(t) g(t)$. Let $V$ be the valuation ring associated with $\partial$, namely, $V=\{r(t) \in F(t) \mid \partial r(t) \geq 0\}$. Note that $F[t], F(t)$ and $V$ are all countable. Consequently, $V$ is a countable-dimensional vector space over $F$. 
Let $v_{0}, v_{1}, \ldots, v_{n}, \ldots$ be a basis of $V$ over $F$. First, we may assume that $\partial v_{i} \neq \partial v_{j}$ for $i \neq j$. Suppose that $n$ is the least integer such that $\partial v_{n}=\partial v_{i}$ for some $i<n$. Choose $\alpha_{i} \in F$ so that $v_{n} / v_{i}$ $\alpha_{i} \in t V$; then $\partial\left(v_{n}-\alpha_{i} v_{i}\right)>\partial v_{i}$. If $\partial\left(v_{n}-\alpha_{i} v_{i}\right)=\partial v_{j}$ for some $j<n$, then $\partial\left(v_{n}-\alpha_{i} v_{i}-\alpha_{j} v_{j}\right)>\partial v_{j}$ for some $\alpha_{j} \in F$. Continuing this process we get a $v_{n}^{\prime}$ such that $\partial v_{n}^{\prime} \neq \partial v_{i}$ for all $i<n$ and that $\left\{v_{0}, v_{1}, \ldots, v_{n-1}, v_{n}^{\prime}\right\}$ spans the same subspace as $\left\{v_{0}, v_{1}, \ldots, v_{n-1}, v_{n}\right\}$ does. Next, we assume, by reordering, that $\partial v_{0}<\partial v_{1}<\partial v_{2}<\cdots$. For $v=\alpha_{k} v_{k}+\alpha_{k+1} v_{k+1}+\cdots$ with $\alpha_{k} \neq 0$, we see that $\partial v=\partial v_{k}$. Since $v_{0}, v_{1}, v_{2}, \ldots$ span the whole space $V$, we must have $\partial v_{0}=0$, $\partial v_{1}=1, \partial v_{2}=2$ and so on.

We begin by constructing a ring which is similar to that in Bergman's example [1; Example 4.26]. Let $S$ be the set of those $x \in E=\operatorname{End}_{F}(V)$ such that $(x-a) t^{n} V=0$ for some $a \in F(t)$ and some nonnegative integer $n$. As in $[1 ;$ p. 47] we observe that $a$ depends only on $x$, that is, for each $x \in S$ there is a unique element $\varphi x \in F(t)$ such that $(x-\varphi x) t^{n} V=0$ for some $n \geq 0$. Also, it can be verified that $S$ is an $F$-subalgebra of $E$ containing $F[t]$ and that $\varphi$ is an $F$-algebra map of $S$ onto $F(t)$. In addition, $\operatorname{ker} \varphi$ is a regular ideal of $S$ and $S / \operatorname{ker} \varphi \simeq F(t)$, and therefore $S$ is a regular ring. However, $S$ is not unit-regular because of the existence of $t \in S$ which is injective but not surjective on $V$.

Let us fix a basis $v_{0}, v_{1}, v_{2}, \ldots$ of $V$ over $F$ with $\partial v_{n}=n$ for all $n$. Then $v_{n}, v_{n+1}, \ldots$ form a basis of $t^{n} V$ over $F$. Let $\pi_{n}$ be the projection of $V$ onto the subspace spanned by $v_{0}, v_{1}, \ldots, v_{n}$ with kernel $t^{n+1} V$. Consider the matrix of $a \in S$ with respect to the basis $v_{0}, v_{1}, v_{2}, \ldots$ Certainly, it is column-finite. That is, for any $m \geq 0$ there exists $n \geq 0$ such that $\left(1-\pi_{n}\right) a \pi_{m}=0$. Also, it is row-finite: for any $m \geq 0$ there exists $n \geq 0$ such that both $(a-\varphi a) t^{n} V=0$ and $(\varphi a) t^{n} V \subseteq t^{m+1} V$, consequently, $a\left(t^{n} V\right) \subseteq t^{m+1} V$ and $\pi_{m} a\left(1-\pi_{n}\right)=0$.

Set $W=S \times \prod_{k=0}^{\infty} \pi_{k} E \pi_{k}$ and write elements of $W$ as sequences $w=\left(w_{-1}, w_{0}, w_{1}, \ldots\right)$ where $w_{-1} \in S$ and $w_{k} \in \pi_{k} E \pi_{k}$ for $k \geq 0$. Let $R$ be the set of elements $w \in W$ satisfying the following two conditions: (i) for any $m \geq 0$ there exists $n \geq 0$ such that $w_{k} \pi_{m}=$ $w_{-1} \pi_{m}$ for all $k \geq n$; (ii) for any $m \geq 0$ there exists $n \geq 0$ such that $\pi_{m} w_{k}=\pi_{m} w_{-1}$ for all $k \geq n$. It is clear that $R$ is an $F$-subspace of $W$. To show that $R$ is a ring, we consider any $u, w \in R$ and $m \geq 0$. There exists $n \geq 0$ such that $w_{k} \pi_{m}=w_{-1} \pi_{m}$ for all $k \geq n$. Now because $w_{-1} \in S$ is column-finite, $w_{-1} \pi_{m}=\pi_{j} w_{-1} \pi_{m}$ for some $j \geq 0$. Also, there exists $n^{\prime} \geq 0$ such that $u_{k} \pi_{j}=u_{-1} \pi_{j}$ for all $k \geq n^{\prime}$. Then 
$u_{k} w_{k} \pi_{m}=u_{k} w_{-1} \pi_{m}=u_{k} \pi_{j} w_{-1} \pi_{m}=u_{-1} \pi_{j} w_{-1} \pi_{m}=u_{-1} w_{-1} \pi_{m}$ for all $k \geq \max \left\{n, n^{\prime}\right\}$. Similarly, we can show that there exists $n^{\prime \prime} \geq$ 0 such that $\pi_{m} u_{k} w_{k}=\pi_{m} u_{-1} w_{-1}$ for all $k \geq n^{\prime \prime}$. Thus, $u w \in R$. Therefore $R$ is an $F$-subalgebra of $W$.

Let $\alpha: R \rightarrow \prod_{k=0}^{\infty} \pi_{k} E \pi_{k}$ be the projection $\left(w_{-1}, w_{0}, w_{1}, \ldots\right) \mapsto$ $\left(w_{0}, w_{1}, \ldots\right)$. Given any $w \in \operatorname{ker} \alpha, w_{k}=0$ for all $k \geq 0$. For any $m \geq 0$ we have $w_{-1} \pi_{m}=w_{k} \pi_{m}=0$ for some $k$. Hence, $w_{-1}=0$ and so $w=0$. Thus $\alpha$ is injective. If $w_{k} \in \pi_{k} E \pi_{k}, k=0,1, \ldots, n$, then $w=\left(0, w_{0}, w_{1}, \ldots, w_{n}, 0, \ldots\right) \in R$ and $\alpha w=\left(w_{0}, w_{1}, \ldots, w_{n}, 0, \ldots\right)$. In other words, $\bigoplus_{k=0}^{\infty} \pi_{k} E \pi_{k} \subseteq \alpha R$.

Let $\beta: R \rightarrow S$ be the projection $\left(w_{-1}, w_{0}, w_{1}, \ldots\right) \mapsto w_{-1}$. For $x \in$ $S$, set $w=\left(x, \pi_{0} x \pi_{0}, \pi_{1} x \pi_{1}, \ldots\right) \in W$. Let $m \geq 0$. Since $x$ is columnfinite, there exists $n \geq 0$ such that $\left(1-\pi_{k}\right) x \pi_{m}=0$ for all $k \geq n$. Then $w_{k} \pi_{m}=\pi_{k} x \pi_{k} \pi_{m}=\pi_{k} x \pi_{m}=x \pi_{m}=w_{-1} \pi_{m}$ for all $k \geq \max \{m, n\}$. Similarly, there exists $n^{\prime} \geq 0$ such that $\pi_{m} w_{k}=\pi_{m} w_{-1}$ for all $k \geq n^{\prime}$. Thus $w \in R$ and $\beta w=x$. Hence, $\beta$ is surjective.

It remains to show that $R$ is regular. But since $R / \operatorname{ker} \beta \simeq S$ is regular, it suffices to show the regularity of $\operatorname{ker} \beta$. Let $w \in \operatorname{ker} \beta$. For each $m \geq 0$ there exist $n_{m} \geq 0$ such that $w_{k} \pi_{m}=\pi_{m} w_{k}=0$ for all $k \geq$ $n_{m}$. Without loss of generality, we may assume that $0<n_{0}<n_{1}<\cdots$. For $0 \leq k<n_{0}$, choose $u_{k} \in \pi_{k} E \pi_{k}$ such that $w_{k} u_{k} w_{k}=w_{k}$. For $n_{m} \leq k<n_{m+1}$, we have $w_{k} \in\left(1-\pi_{m}\right) \pi_{k} E \pi_{k}\left(1-\pi_{m}\right)$, and so choose $u_{k} \in\left(1-\pi_{m}\right) \pi_{k} E \pi_{k}\left(1-\pi_{m}\right)$ such that $w_{k} u_{k} w_{k}=w_{k}$. Thus $u=$ $\left(0, u_{0}, u_{1}, \ldots\right) \in W$ and $w u w=w$. Moreover, $u_{k} \pi_{m}=\pi_{m} u_{k}=0$ for all $k \geq n_{m}$ by construction. Hence, $u \in R$ and so $u \in \operatorname{ker} \beta$. Therefore, $\operatorname{ker} \beta$ is regular, and so $R$ is regular. On the other hand, $S$, which is not unit-regular, is a homomorphic image of $R$. Consequently, $R$ cannot be unit-regular.

Thus, we have constructed a regular ring $R$ which is not unit-regular. Since $\bigoplus_{k=0}^{\infty} \pi_{k} E \pi_{k} \subseteq \alpha R \subseteq \prod_{k=0}^{\infty} \pi_{k} E \pi_{k}$, where $\alpha$ is a monomorphism and $\pi_{k} E \pi_{k} \simeq M_{k}(F), R$ is a subdirect product of simple artinian rings. This settles Question 6 in the negative.

A ring $R$ is said to be of bounded index if there exists a positive integer $n$ such that $x^{n}=0$ for all nilpotent elements $x$ in $R$. The seventh question is: If $R$ is a regular ring such that every nonzero twosided ideal of $R$ contains a nonzero two-sided ideal of bounded index, is $R$ unit-regular? This question is in fact equivalent to Question 6. Instead of showing this, one can verify easily that the example constructed above satisfies the condition of this question. Let $I$ be a nonzero two-sided ideal of $\alpha R$. Let $w=\left(w_{0}, w_{1}, w_{2}, \ldots\right) \in I$ with 
$w_{n} \neq 0$ for some $n \geq 0$. Since $\bigoplus_{k=0}^{\infty} \pi_{k} E \pi_{k} \subseteq \alpha R$ and $\pi_{n} E \pi_{n}$ is simple, it follows that $I$ contains a nonzero two-sided ideal isomorphic to $\pi_{n} E \pi_{n}$ which is clearly of bounded index. This gives a negative answer to Question 7.

A pseudo-rank function on a regular ring $R$ is a map $N: R \rightarrow[0,1]$ such that (a) $N(1)=1$, (b) $N(x y) \leq \min \{N(x), N(y)\}$ for all $x, y \in R$, (c) $N(e+f)=N(e)+N(f)$ for all orthogonal idempotents $e, f \in R$. If, in addition, $N(x)=0$ only if $x=0, N$ is called a rank function on $R$. The set of all pseudo-rank functions on $R$ is denoted by $\mathbf{P}(R)$. Given a family $X \subseteq \mathbf{P}(R)$, we use $\operatorname{ker}(X)$ to denote the kernel of $X$, namely, $\operatorname{ker}(X)=\{x \in R \mid N(x)=0$ for all $N \in X\}$. Since all simple artinian rings have rank functions [1; Corollary 16.6], then $\sum_{k=0}^{\infty}\left(1 / 2^{k+1}\right) N_{k}$ defines a rank function on $\prod_{k=0}^{\infty} M_{k}(F)$, where $N_{k}$ is a rank function on $M_{k}(F)$. Thus any regular subdirect product $R$ of $\prod_{k=0}^{\infty} M_{k}(F)$ has a rank function and hence $\operatorname{ker}(\mathbf{P}(R))=0$. Therefore we have obtained a counterexample to the eighth question: If $R$ is a regular ring such that $\operatorname{ker}(\mathbf{P}(R))=0$, is $R$ unit-regular? Since a regular ring with a rank function contains no infinite direct sums of nonzero pairwise isomorphic right or left ideals [1; Proposition 16.11], the second part of Question 9 is also settled: If $R$ is a regular ring which contains no infinite direct sums of nonzero pairwise isomorphic right ideals, is $R$ unit-regular? Finally, a regular ring with a rank function satisfies the hypothesis of Question 11 [3; Theorem 5]: Let $R$ be a regular ring, and assume that whenever $x, y \in R$ such that $x y=y x$ and $x R+y R=R$, then $R x+R y=R$. Is $R$ unit-regular? Thus our example also provides a negative answer to this question.

Acknowledgment. This is a revised version of our original paper. The referee pointed out that the technique of D. V. Tyukavkin in [4; proof of Theorem 2] which was not known to us then can streamline our proof considerably. He also indicated the existence of rank function in our example which enables us to settle Questions 8, 9 (second part) and 11 also in the negative.

\section{REFERENCES}

[1] K. R. Goodearl, Von Neumann Regular Rings, Pitman, London, 1979.

[2] K. R. Goodearl and P. Menal, Stable range one for rings with many units, J. Pure Applied Algebra, 54 (1988), 261-287.

[3] D. Handelman and R. Raphael, Regular Shur rings, Arch. Math., 31 (1978), 332-338. 
[4] D. V. Tyukavkin, Regular rings with involution, Vestnik Moskov. Univ. Ser. I Mat. Meh., 39 (3) (1984), 29-32. (English translation: Moscow Univ. Math. Bull., 39 (3) (1984), 38-41.)

Received January 10, 1988.

National TAIWAN University

TAIPEI, TAIWAN 10674 



\section{PACIFIC JOURNAL OF MATHEMATICS EDITORS}

\author{
V. S. VARADARAJAN \\ (Managing Editor) \\ University of California \\ Los Angeles, CA 90024-1555-05 \\ Herbert Clemens \\ University of Utah \\ Salt Lake City, UT 84112 \\ ThOMAS ENRIGHT \\ University of California, San Diego \\ La Jolla, CA 92093
}

\section{R. FINN}

Stanford University

Stanford, CA 94305

HeRmann FlaschKa

University of Arizona

Tucson, AZ 85721

VAUGHAN F. R. JONES

University of California

Berkeley, CA 94720

Steven KerckhofF

Stanford University

Stanford, CA 94305

\author{
RobION KIRBY \\ University of California \\ Berkeley, CA 94720 \\ C. C. Moore \\ University of California \\ Berkeley, CA 94720 \\ HAROLD STARK \\ University of California, San Diego \\ La Jolla, CA 92093
}

\section{ASSOCIATE EDITORS}
R. ARENS
E. F. BECKenBach
B. H. NeumanN
F. WOLF
K. YoshidA (1906-1982)
(1904-1989)

\section{SUPPORTING INSTITUTIONS}

UNIVERSITY OF ARIZONA

UNIVERSITY OF BRITISH COLUMBIA

CALIFORNIA INSTITUTE OF TECHNOLOGY

UNIVERSITY OF CALIFORNIA

MONTANA STATE UNIVERSITY

UNIVERSITY OF NEVADA, RENO

NEW MEXICO STATE UNIVERSITY

OREGON STATE UNIVERSITY
UNIVERSITY OF OREGON

UNIVERSITY OF SOUTHERN CALIFORNIA

STANFORD UNIVERSITY

UNIVERSITY OF HAWAII

UNIVERSITY OF TOKYO

UNIVERSITY OF UTAH

WASHINGTON STATE UNIVERSITY

UNIVERSITY OF WASHINGTON 


\section{Pacific Journal of Mathematics}

Vol. 142, No. $1 \quad$ January, 1990

Marco Andreatta, Mauro Beltrametti and Andrew Sommese, Generic properties of the adjuction mapping for singular surfaces and applications

Chen-Lian Chuang and Pjek-Hwee Lee, On regular subdirect products of

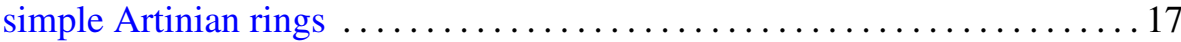

Fernando Giménez and Vicente Miquel Molina, Volume estimates for real hypersurfaces of a Kaehler manifold with strictly positive holomorphic sectional and antiholomorphic Ricci curvatures $\ldots \ldots \ldots \ldots \ldots \ldots 23$

Richard J. Griego and Andrzej Korzeniowski, Asymptotics for certain Wiener integrals associated with higher order differential operators

Abdeslam Mesnaoui, Unitary bordism of classifying spaces of quaternion

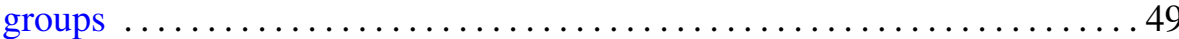

Abdeslam Mesnaoui, Unitary cobordism of classifying spaces of quaternion

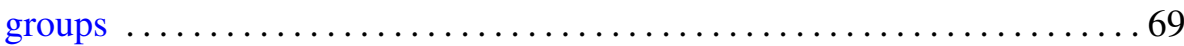

Jesper M. Møller, On equivariant function spaces $\ldots \ldots \ldots \ldots \ldots \ldots \ldots \ldots$

Bassam Nassrallah, A $q$-analogue of Appell's $F_{1}$ function, its integral representation and transformations

Peter A Ohring, Solvability of invariant differential operators on metabelian

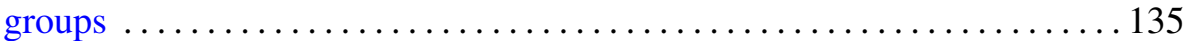

Athanase Papadopoulos and R. C. Penner, Enumerating pseudo-Anosov foliations

Ti-Jun Xiao and Liang Jin, On complete second order linear differential

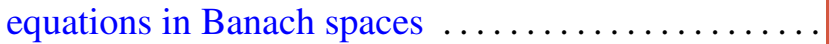

Carl Widland and Robert F. Lax, Weierstrass points on Gorenstein curves 